\title{
SEQUENTIAL SAMPLING OF THE LODGEPOLE NEEDLE MINER ${ }^{1}$
}

\section{R. W. STARK ${ }^{2}$}

\section{INTRODUCTION}

This paper deals with the application of sequential sampling for rapid population estimates of the lodgepole needle miner, Recurvaria milleri Busck. Briefly, it is a sampling plan with no fixed sample size and should be of value to provide reasonable population estimates over large areas with a minimum of time and effort.

\section{Application of the Sequential Plan}

The first requirement is to 'establish the type of distribution. The data used are valley bottom samples from intensive population studies in Yoho National Park ${ }^{2}$. Eight branch tips from each of 40 trees were examined, four from the upper third of the crown and four from the lower third. The unit of measurement chosen for the population estimate was the number of live larvae per branch tip. The branch tips were cut back to include five-year old needles. The Chi-square test indicated that the distribution was normal.

The formulae for sequential analysis applicable to the normal distribution were obtained ${ }^{3}$ and two sets of lines were formulated. Two sets of lines were required as it was desired to establish three classes of infestation intensity, light, medium, and heavy. These classes are explained in evolving the line formulae, as follows:

$\mathrm{m}_{1}=$ the smaller average $=5$; below 5 per tip will be called a light - infestation.

$\mathrm{m}_{2}=$ the larger average $=15$; above 15 per tip will be called a medium infestation.

$\alpha 1=$ the risk of accepting $m=m_{1}$ when $m=m_{2}$ i.e. calling a medium infestation light. This is more serious than calling a light infestation medium so this risk was set at 5 per cent $=.05$.

$\beta_{1}=$ the risk of rejecting $\mathrm{m}=\mathrm{m}_{1}$ when $\mathrm{m}=\mathrm{m}_{1}$ i.e. calling a light infestation medium. This is less serious than the above so this risk was set at 10 per cent $=.1$.

$\sigma=$ standard deviation for 320 observations taken to establish the normal distribution $=15.62$.

$\mathrm{x}=$ the number of live needle miners per branch tip.

$\Sigma X=$ the cumulative sum of $\mathrm{x}$ 's for $\mathrm{n}$ observations in a sample.

$$
a_{1}=a \text { constant }=\log _{e} \frac{\left(1-\beta_{1}\right)}{\alpha_{1}}=2.89 .
$$

1 Contribution No. 45, Division of Forest Biology. Science Service, Department of Agriculture, Ottawa, Canada.

2 Technical Officer, Laboratory of Forest Zoology, Calgary, Alberta. 


$$
\mathrm{b}_{1}=\mathrm{a} \text { constant }=\log _{\mathrm{e}} \frac{\left(1-\alpha_{1}\right)}{\beta_{1}}=2.25 .
$$

The lines are:

$$
\begin{aligned}
& \text { (1) } \sum X=-h_{1}+s n \\
& \text { (2) } \Sigma X=h_{2}+s n
\end{aligned}
$$

where:

$$
\begin{aligned}
& h_{1} \text { and } h_{2} \text { are the intercepts with the } \Sigma X \text { axis and are equal to: } \\
& h_{1}=\frac{b \sigma^{2}}{m_{2}-m_{1}}=54.93 \quad h_{2}=\frac{a \sigma^{2}}{m_{2}-m_{1}}=70.53
\end{aligned}
$$

and: $s=$ slope of the lines $=\frac{m_{1}+m_{2}}{2}=10$

Therefore the formulae for the first set of lines are:

$$
\begin{aligned}
& \text { (1) } \Sigma_{1} X=-54.93+10 \mathrm{n} \\
& \text { (2) } \Sigma_{\mathrm{X}}=70.53+10 \mathrm{n}
\end{aligned}
$$

The second set of lines was formulated using the following values:

$$
\begin{aligned}
& \mathrm{m}_{3}=25 ; \text { below } 25 \text { per tip will be called a medium infestation } \\
& \mathrm{m}_{4}=35 ; \text { above } 35 \text { per tip will be called a heavy infestation } \\
& \alpha_{2}=\beta_{2}=5 \text { per cent }=.05 \\
& \mathrm{a}_{2}=\mathrm{b}_{2}=2.94 \\
& \mathrm{~h}_{3}=\mathrm{h}_{4}=71.8 \\
& \quad \mathrm{~s}=30
\end{aligned}
$$

The formulae for the second set of lines are:

(3) $\Sigma X=-71.8+30 n$

These lines were then plotted with $\Sigma \mathrm{X}$ on the ordinate and $\mathrm{n}$ on the abscissa (Plate 1).

\section{Discussion}

In sampling needle miner populations by this method certain precautions must be observed. It has been proved that there is a significant difference between the two crown levels in degree of infestation i.e. the upper third and the lower third of the crown ${ }^{2}$. Therefore, the crowns must be sampled separately if a true picture of the infestation is to be obtained. Sampling can be done in three ways:

1. By taking alternate samples from the upper and lower crowns, which will give an average for the whole tree or group of trees. However, this will be lower than the upper crown figures alone.

2. By sampling upper crown alone and/or

3. By sampling lower crown alone.

It is necessary for the sampler to indicate from which part of the tree his samples were taken. No matter which of the above methods is followed the procedure in the use of the graph is the same. Sampling is continued 


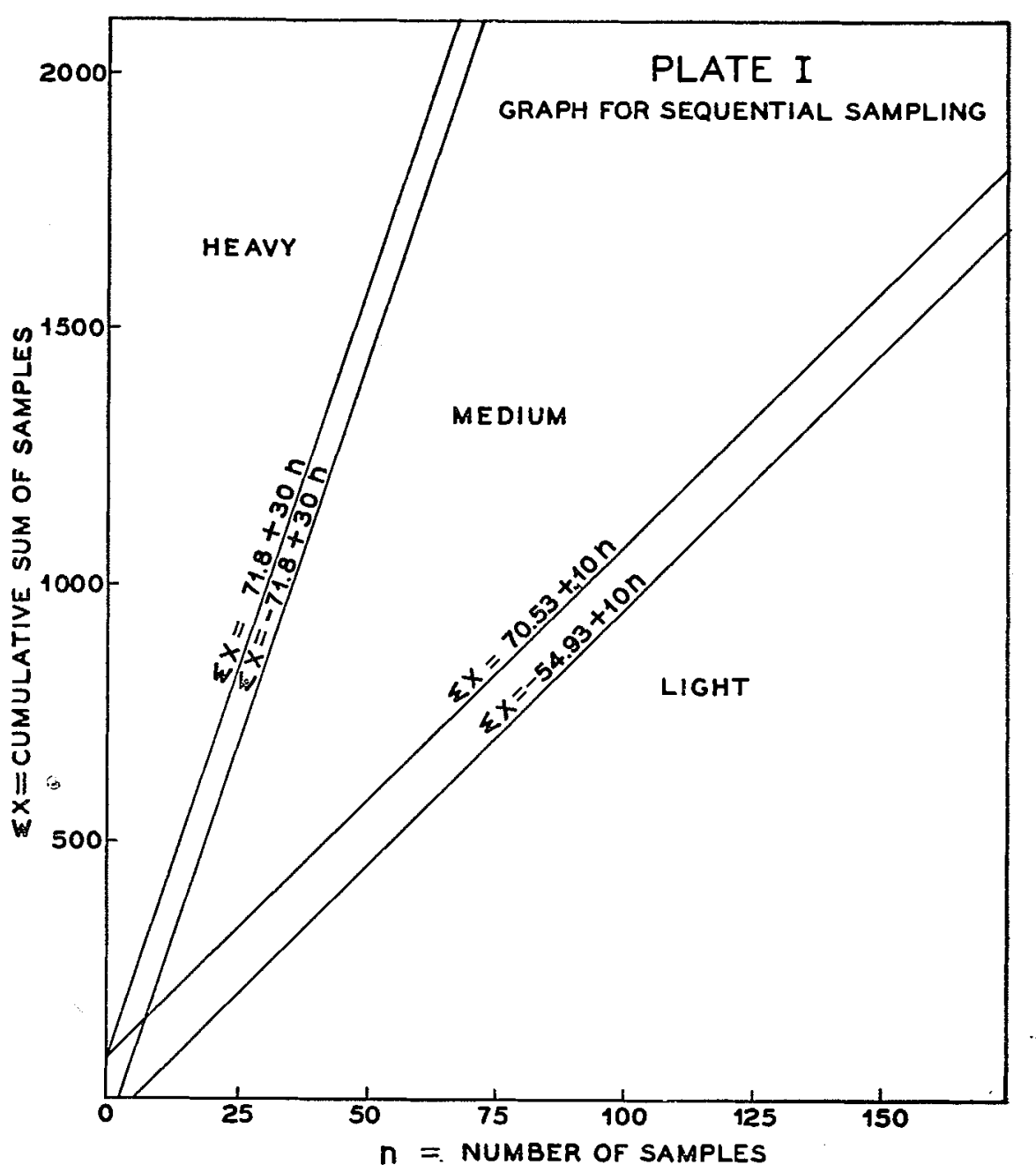

until the cumulative sum of the observations (number of miners per tip) when plotted, crosses one of the four lines. Thus it is known within an error of five per cent (10 per cent if light) into which of the infestation classes the population which is being sampled belongs.

As there is no fixed sample size in this method, no set rules can be laid down for the number of trees to be sampled. It has been demonstrated ${ }^{2}$ that sampling a greater number of branch tips from fewer trees will obtain the same results as fewer branch tips from more trees. The former method, however, requires a greater number of branch tips to arrive at an estimate. It is 
suggested in applying this method that four or eight branch tips be taken from each crown level in each tree until an answer is obtained.

The extensive stands of pure lodgepole pine susceptible to needle miner outbreaks have been pointed out by MacLeod ${ }^{1}$. Laboratory staffs cannot hope to investigate all areas thoroughly, but with this simple chart any forest worker can obtain a rapid estimate of a needle miner population. This will materially reduce the time spent in investigating areas that are below 'serious' proportions and will permit a greater sample coverage than with previous methods.

\section{SUMMARY}

Sequential sampling is applicable to needle miner populations. Three grades of infestation intensity are delimited. The cumulative sum of observations i.e. numbers of miners per branch tip is plotted on a prepared chart. When the plotted points cross one of the four lines it is established within a set error limit to which grade of infestation the outbreak belongs. This method of classifying needle miner populations has an important place in preliminary surveys of needle miner outbreak areas, allowing the direction of most effort to those areas warranting it.

\section{ACKNOWLEDGMENTS}

G. B. Oakland, Head of the Biometric Unit, Science Service, Ottawa, suggested the possibility of applying sequential sampling to needle miner populations and assisted in the analysis and presentation of the data. His many contributions are gratefully acknowledged.

\section{Literature Cited}

1. MacLEOD, J. H. Notes on the Lodgepole Needle Miner. Recurvaria milleri Busck (Lepidoptera: Gelechiidae) and its Parasites in Western North America. Canadian Ent. 83. 295-301. 1951.

2. STARK, R. W. Population Studies of the Lodgepole Needle Miner. In Press. 1952.

3. Various Authors. Sequential Analysis of Statistical Data: Applications. Columbia Research Group GRG 255 (rev.) AMP Report 30.2 (rev.) 1951. 